\title{
Resolution enhancement of thermal images in active dynamic thermography sequences
}

by M. Kaczmarek*, M. Borwanski*

\author{
*Dept. of Biomedical Engineering, Gdansk University of Technology, Narutowicza 11/12, 80-233 Gdansk, \\ Poland, mariusz.kaczmarek@biomed.eti.pg.gda.pl
}

\begin{abstract}
Recording time series of thermograms allows to reconstruct a thermal images of better spatial resolution and then to calculate basing on new high resolution (HR) sequence parametric images in Active Dynamic Technique. It is quite important because of low original spatial resolution of the detector in most of thermal cameras which are used today. Modification of equipment and reconstructive algorithms for HR procedure are discussed in the paper. Also clinical case of burn diagnosis using HR ADT technique is shown.
\end{abstract}

\section{Introduction}

The concept of Infrared Non-Destructive Testing (IR/NDT) has been known in industry for several years [1,2]. Active Dynamic Thermography (ADT), based on analysis of thermal transients at the surface of a tested region after external pulse excitation, may be regarded as an advanced version of IR/NDT suitable for medical applications [3]. In ADT the analysis of heat transfer in time enables determination of thermal time constants, which are dependent on such thermo-physical biomaterial properties as thermal diffusivity and conductivity as well as geometry of a region of interest (ROI). Strong correlation of those measurable parameters with the state of a tested organ allows determination of it's sub-surface structure. IR thermography instrumentation and application of dedicated data processing for analysis of thermal transients after external thermal excitations allows to get objective, quantitative thermal data of tested tissues [3], therefore it has potential value as an tool for microstructure changes recognition in tissues. Idea of super resolution reconstruction was early presented for visual photos and video sequences, eg. [4]. Also in thermal technique that was attempt to involve such technique with dual camera system [5]. Here we present one camera system with special rotating device for motion blur generating.

\section{Methods}

The precise detection of relative movement between the registered image is an element common to most algorithms Super-Resolution and has a significant impact on the outcome of their actions. Quality of reconstructed highresolution image is directly related to the accuracy of the shift detection.

The issue is quite complicated since the search shifts are not integers - they are fractions of a pixel (called subpixel shift). This follows from the basic assumptions for Super-Resolution method - only when the images in the sequence are shifted by a fractional (incomplete) part of pixel it is possible to reconstruct an image with higher resolution. Frames transferred by the total number of pixel not contribute additional information. Described algorithms and methods are implemented in HR Thermography application. GUI of application is presented on fig. 1.

\subsection{Shift estimation algorithms}

Frequency methods are applicable only to a relatively simple model of motion - shifts are global (affecting the entire image) and planar (occurs only in a plane parallel to the plane of the image). One can distinguish vertical displacement, horizontal, rotation and scaling. Their advantage is that the above-mentioned phenomena can be relatively easy to describe in the frequency domain. Distortions arising from the occurrence of aliasing can be much easier to determine than the mathematical methods in the field of space. Implementation is often relatively simple and the computational complexity is very different for the different algorithms. It depends on the adopted mathematical model and the required precision. However, you can generally assume that the simple method of frequency are generally faster and requires less expensive hardware than methods basing on spatial domain.

Spatial methods allow the use of much more complex models of motion and detection of transfers in respect of local elements in the picture (detected as a result of various detection algorithms such as the face). Often these are iterative methods, involving the following steps to minimize the estimation error (defined for the assumed model). They are very commonly used among others in the algorithms to track the movement of objects.

Motion model adopted in this work for the image reconstruction method for Super-Resolution is a simple planar model. Search shifts are global and consist in the translation, which is possible to type a vertical and horizontal displacement. In the initial phase of the project was taken into account the rotation, but in the course of verifying the effectiveness of different algorithms on data from thermography camera was rejected. The obtained values of relative 
rotation between the two images were on the computational error limits. For this reason and given the very small probability of the test sequences rotation phenomenon, this effect was omitted.

In the adopted variant of the key differences between the tested algorithms, and thus the criteria for selecting the method best suited for use with thermal images, the complexity of implementation, speed and above all, accuracy of estimation. Precision is related to the characteristics of the examined images. Different methods have different resistance to specific types of distortion and noise in the test images. Went to the final application, three different algorithms - two using Fourier transform and one operating in the field of space - using a Taylor series.

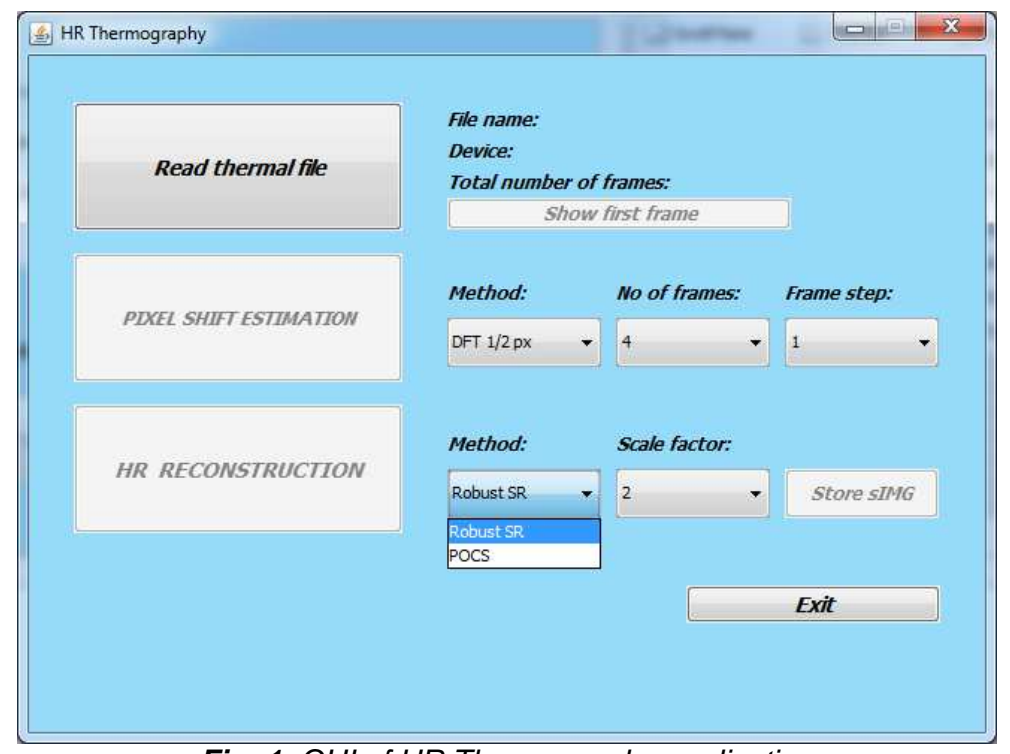

Fig. 1. GUI of HR Thermography application

\subsubsection{Frequency Vandewelle method}

This is a frequency domain method, proposed by $P$. Vandewalle [6]. It allows independent determination of the horizontal displacement $(\Delta \mathrm{x} 1)$ and vertical $(\Delta \mathrm{x} 2)$ and rotation $(\Delta \varphi)$ with respect to an axis perpendicular to the plane of the image. Also takes into account the occurrence of aliasing of input images.

Estimation of the transfers between individual frames of the test sequence is based on the claims of displacement (called shift theorem) for the Fourier transform in terms of two-dimensional. Moving in a plane parallel to the plane of the image can be represented in the field of Fourier, as a linear phase shift. Assuming a reference image $f_{1}(\mathbf{x})$ and its shifted version $f_{2}(\mathbf{x})$ :

$$
f_{2}(\mathbf{x})=f_{1}(\mathbf{x}+\Delta \mathbf{x})
$$

Fourier transform may be described as follows:

$$
F_{2}(u)=\iint_{\mathbf{x}} f_{2}(\mathbf{x}) \cdot e^{-j 2 \pi u^{T} \mathbf{x}} d \mathbf{x}=\iint_{\mathbf{x}} f_{1}(\mathbf{x}+\Delta \mathbf{x}) \cdot e^{-j 2 \pi u^{T} \mathbf{x}} d \mathbf{x}=e^{j 2 \pi u^{T} \Delta \mathbf{x}} \iint_{\mathbf{x}} f_{1}\left(\mathbf{x}^{\prime}\right) \cdot e^{-j 2 \pi u^{T} \mathbf{x}^{\prime}} d \mathbf{x}^{\prime}=e^{j 2 \pi u^{T} \Delta \mathbf{x}} F_{1}(u)
$$

$F_{1}(u)$ and $F_{1}(u)$ are the Fourier transform of respectively reference image and shifted image, $\mathbf{x}^{\prime}-$ transformed coordination.

The parameters describing the displacement $\Delta \mathbf{x}$ can be calculated as the slope of the phase difference $F_{1}(u)$ and $F_{1}(u)$. The least squares method is used for this purpose.

If the input image sequence is also aliasing, detection becomes much less precise. To mitigate this effect, the authors propose to use to calculate the parameters of offset only part of the spectrum, which contains low frequencies. With proper selection of low-pass filter is free of aliasing and does not contain the high-frequency noise, which has a beneficial effect on the outcome of the estimation.

\subsubsection{Frequency DFT method}

It is a frequency method, the calculation of the Fourier transform is used. It is possible to detect the horizontal and vertical shift in the global context, rotation is ignored. In this work the implementation of two-dimensional DFT transform, based on matrix multiplication is used [7]. The first step is calculated a position with a lower accuracy - to one or half a pixel. This does not require large computational resources. Then the calculation will be repeated with much greater 
accuracy, but only within the immediate neighborhood the result obtained in the first step - an area of size $1.5 \times 1.5$ pixel. After this operation, with the implementation of the DFT matrix, is given an array of sizes $1.5 \mathrm{k}$ to $1.5 \mathrm{k}$, where $\mathrm{k}$ is the rate of increase in precision. Location of maximum in this area is the results, obtained with the required accuracy. Assuming that $\mathrm{k}$ is smaller than the dimensions of the test image, this method is much more efficient than the standard approach. It is possible to estimate with accuracy required for the reconstruction of Super-Resolution, using conventional PC computers.

\subsubsection{Iterative Taylor series method}

Unlike the previous two, it is a method of operating in a spatial domain. The algorithm was described by D. Karen, et. al. [8] in context of an image sequence improvement. This method allows the estimation of the relative horizontal and vertical displacement and rotation. Estimation of all three parameters are the same time. Calculations are performed in image space - $x$, y If $g_{r}(x, y)$ is the reference image and $g_{k}(x, y)$ of the test image from the sequence, which we shift register, the relationship between the position of pixels in these frames can be saved using the following formula:

$$
g_{k}(x, y)=g_{r}(x \cdot \cos (\Delta \theta)-y \cdot \sin (\Delta \theta)+\Delta x, y \cdot \cos (\Delta \theta)-x \cdot \sin (\Delta \theta)+\Delta y)
$$

Shifts horizontal, vertical and rotation are marked sequentially, $\Delta \mathrm{x}, \Delta \mathrm{y}$ and $\Delta \theta$. By the approximation of the values $\sin (\Delta \theta)$ and $\cos (\Delta \theta)$ using them to develop into a Taylor series of first-degree equation is simplified to the form:

$$
g_{k}(x, y) \approx g_{r}(x \cdot-y \cdot(\Delta \theta)+\Delta x, y \cdot+x \cdot(\Delta \theta)+\Delta y)
$$

and follows Taylor series:

$$
g_{k}(x, y) \approx g_{r}(x, y)+(-y \cdot(\Delta \theta)+\Delta x) \cdot \frac{\partial g_{r}}{\partial x}+(x \cdot(\Delta \theta)+\Delta y) \cdot \frac{\partial g_{r}}{\partial y}
$$

It is assumed that the displacements $\mathrm{x}, \mathrm{y}, \mathrm{d}$ remained constant within a certain area. In the general case can be studied full picture - according to the accepted model of the movement. Estimation accuracy can be increased by smoothing first these pictures, such as using low-pass Gaussian filter. This reduces the amount of noise and aliasing effects.

\subsection{HR image reconstruction algorithms}

In the literature has been described many different methods of image reconstruction called Super or HighResolution. A clear majority of current algorithms operating in image space. Frequency methods are commonly found in early publications. Their great advantage is easy implementation and low hardware requirements. Unfortunately they can not be used to more complex models of observation and, therefore, in later work a lot more attention is paid to methods of spatial domain. In this paper we have examined two methods of reconstruction, both belonging to the group of spatial algorithms [9].

\subsubsection{POCS method}

Projection Onto Convex Sets - this is an iterative method to include the reconstruction process prior knowledge of the solution. The main advantages of the method are relatively simple implementation and use very general model of observations. The disadvantages include the ambiguity of the solution (there may be some thugs that match the given conditions) and the relatively high computational complexity. In this paper we used was a variant of this method, which developed Papoulis and Gerchberg [10].

\subsubsection{Robust SR method}

Under the name shown above, there are several methods that differ slightly principle of action. All are designed to get maximum possible resistance to external factors hindering the correct reconstruction. These factors include incorrect estimation of the offsets between frames, inaccurate modeling of the blur and other distortion and moving objects within the recorded sequence. In this paper a method has been implemented, which compiled and published Zomet Assaf, et. al.[11]. It is an iterative procedure, using a median estimator. It is used to reject values that are not compatible with the chosen model representation.

\subsection{Thermal Imaging Technique}

We apply external pulse excitation lasting several seconds, using apparatus for cryotherapy for cooling or air conditioning device. IR camera is applied for capturing of series of thermal images allowing measurements of temperature transients on the tested surface. It is needed high speed image capturing, so sequences were recorded of 60 images per second.

The IR-camera Flir A320G of $0.08^{\circ} \mathrm{C}$ resolution was used for capturing sequences of thermal images. It was placed at the distance of $0.75 \mathrm{~m}$ from the object, in the plane perpendicular to it. The ADT experiment was performed using cooling energy. Adapted for medical use air conditioner device was used as the cooling device, generating mixture 
of air stream of the temperature $15^{\circ} \mathrm{C}$; timing - about 60 second of stimulus and 180 second of recording temperature at the recovery phase (self re-warming). To ensure the generation of thermal camera shake camera mounted on the housing was controlled by a stepper motor with a weight. The electric motor was so driven to perform 120 revolutions per second.

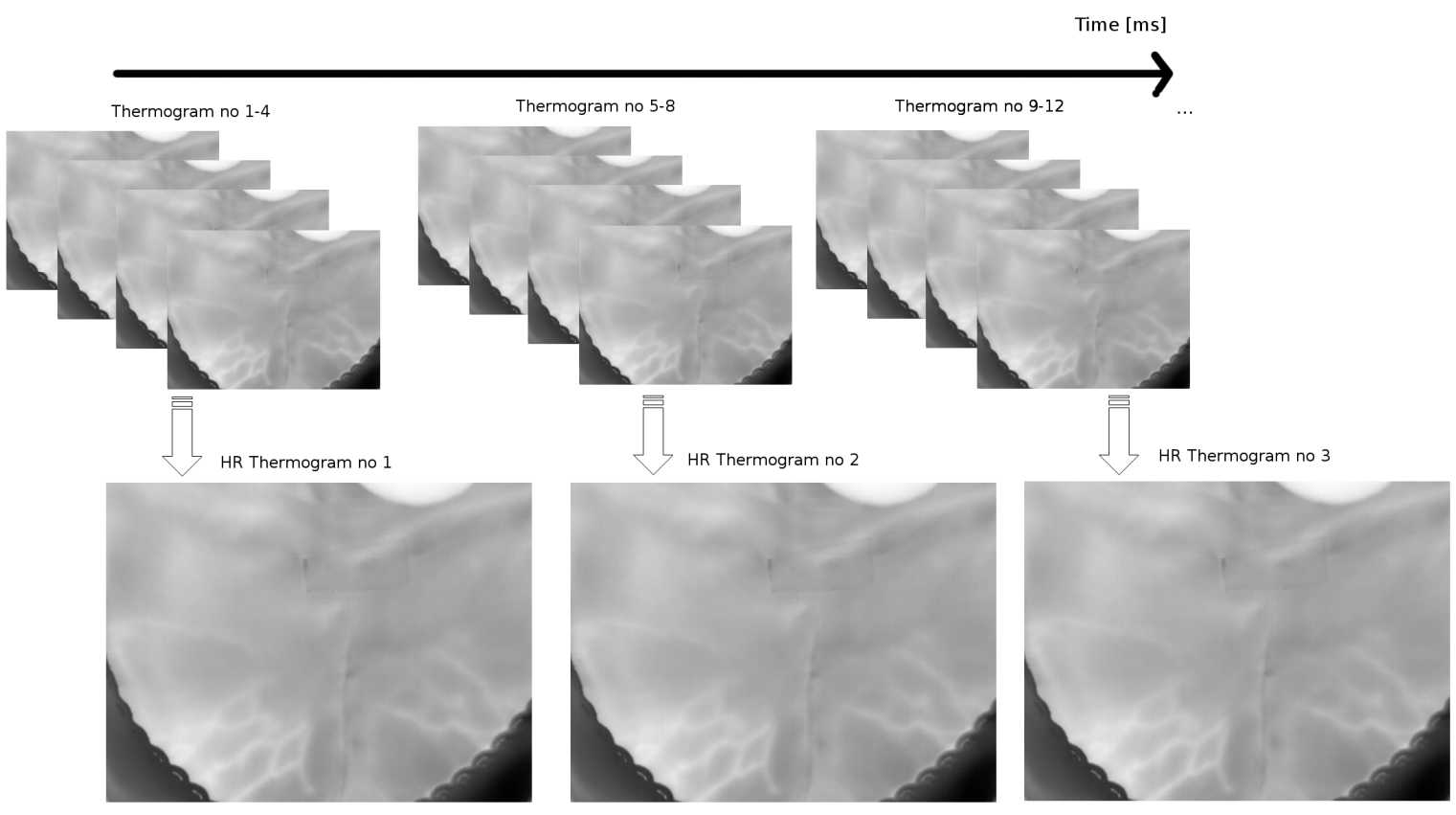

Fig. 2. Procedure for HR reconstruction from thermographic sequence

Recorded sequence is treated in order to determine the sequence of High Resolution thermal images - Fig. 2. This involves the calculation of HR thermogram of each consecutive 4 or 8 frames of thermograms sequence. As a result, we get a sequence of HR thermograms, which is the sequence as if it were recorded at 15 frames per second, or 7 frames per second. This time resolution is quite sufficient for the observed temperature changes in the tissues in ADT technique [4]. Next the HR sequence is passed to the ADT parameters calculation procedures: especially time constant of two exponential model [3].

\section{Results}

Frequency Vandewalle method sometimes gave unlikely results. Although they were not entirely impossible, but clearly different from the values returned by other methods. In the case of a sequence of static occasionally popping up results clearly too big to cattle possible.

DFT method, the frequency response, made the impression of the most reliable. Return values are almost always likely and did not differ much from each other in the sequence recorded in similar conditions. DFT algorithm proved to be the best for the Super-Resolution reconstruction of thermal images. It is of course attention to the fact that this method proved to be the most versatile and resistant to deformation occurring in the test images - as proposed in the literature.

The comparison between the analyzed methods frequency and spatial domain proved that better are algorithms using DFT. Images reconstructed on the basis shifts calculated using this method are characterized by a significantly greater amount of detail and is sharper than it is when using the algorithm of Vandewalle.

Table 1. Results of pixel shift estimation procedure for different algorithms

\begin{tabular}{|c|c|c|c|c|c|c|c|c|}
\hline & \multicolumn{2}{|c|}{ DFT 1/4px } & \multicolumn{2}{c|}{ DFT 1/8px } & \multicolumn{2}{c|}{ Vandewalle } & \multicolumn{2}{c|}{ Iterative Taylor } \\
\hline & $\Delta x$ & $\Delta y$ & $\Delta x$ & $\Delta y$ & $\Delta x$ & $\Delta y$ & $\Delta x$ & $\Delta y$ \\
\hline 1st img & 0.0 & 0.0 & 0.0 & 0.0 & -0.0 & 0.0 & 0.0 & 0.0 \\
\hline 2nd img & 0.0 & -0.25 & -0.125 & -0.125 & -0.2823 & 0.8050 & -0.0442 & -0.1129 \\
\hline 3rd img & -0.25 & -1.0 & -0.125 & -1.0 & 1.4403 & 0.7843 & -0.1287 & -0.1802 \\
\hline 4th img & -0.25 & 0.0 & -0.25 & 0.125 & 1.7580 & 1.7115 & $7.344 E-4$ & -0.2168 \\
\hline
\end{tabular}


Table 1 contains the shift estimation results for the same sequence for the described algorithms. The most realistic estimate was obtained for the DFT 1/8 pixel method which is also confirmed by the subsequent reconstruction of $\mathrm{HR}$ images. The first image is treated as reference image.

As an example of the method selected case patients with hypertrophy scar on the chest. The study sought to determine the ADT thermal parameters for the healthy tissue (skin) and the area of scar tissue. As the scar takes only a few pixels wide, it is important to increase the resolution of a standard thermograms in the sequence.

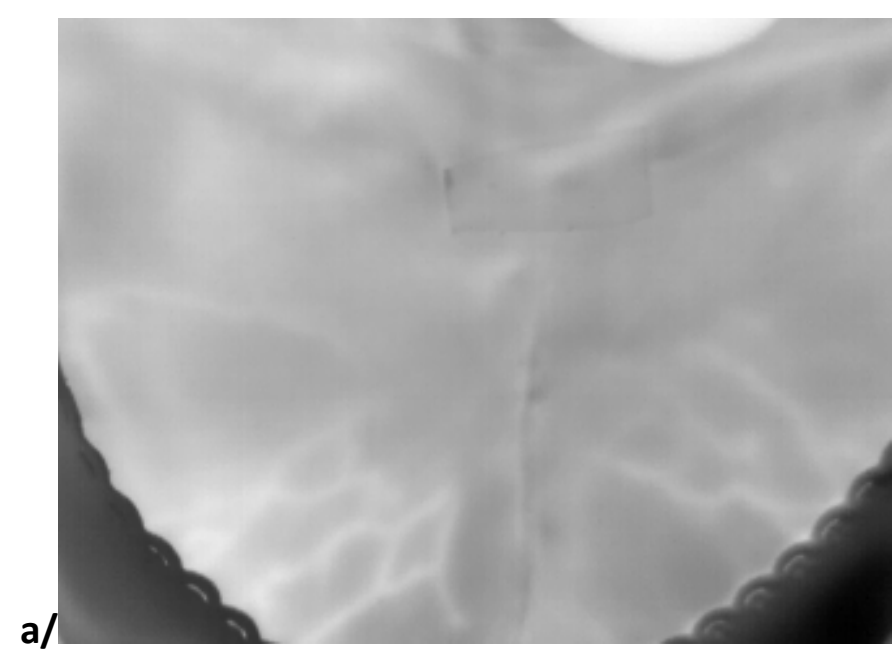

b/

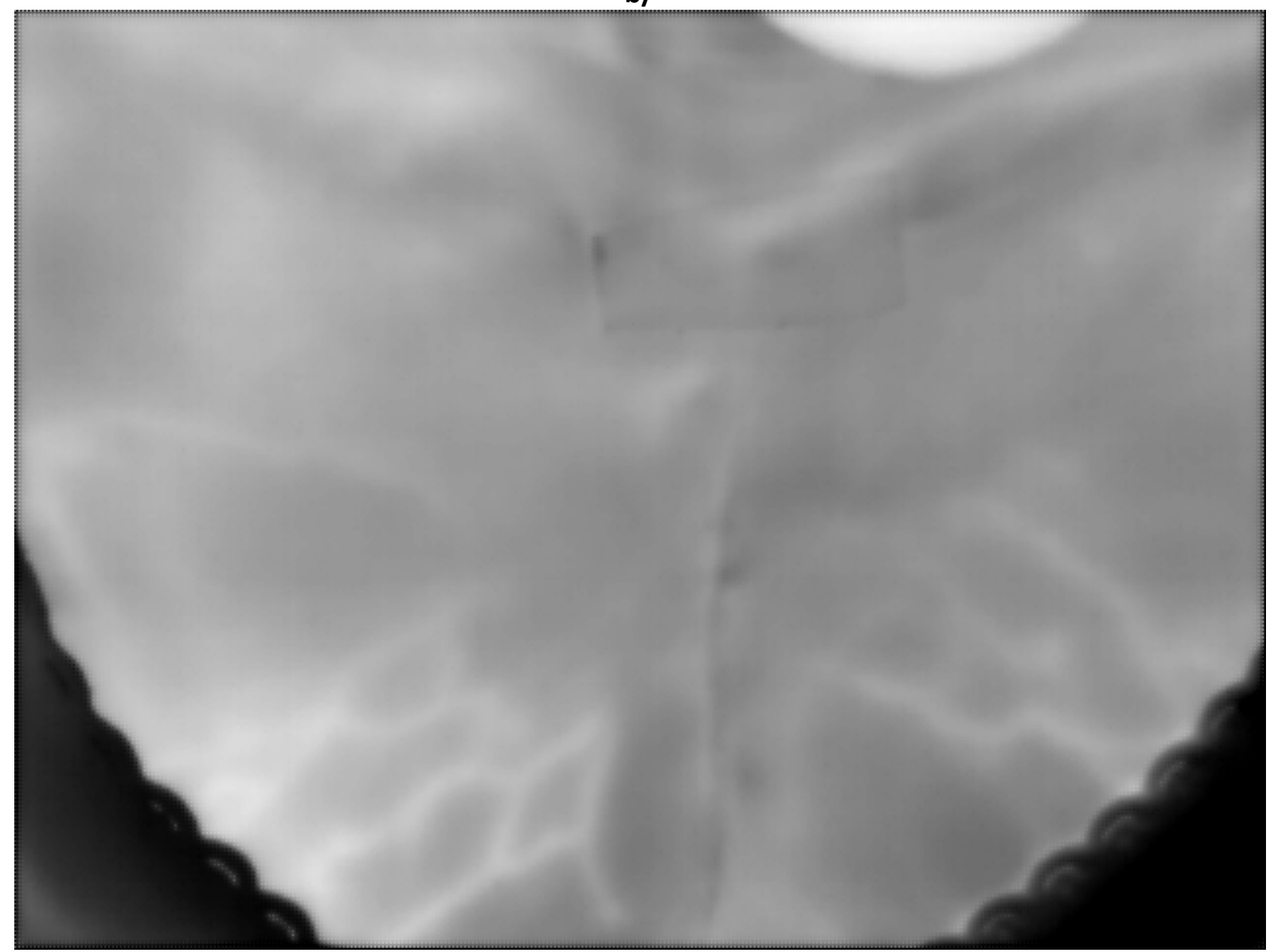

Fig. 3. Example o HR reconstruction; a/ original image, b/ HR reconstruction - $2 x$ increased, 4 frames DFT $1 / 8 p x$ and POCS algorithm 
An important aspect is that the SR method allows to obtain reliable information on the zoomed image. The obtained values of sub-pixels are measured real-life values and not calculated with the use of bilinear interpolation method or bi-cubical. This is particularly important in medical applications of thermography.

\section{Disscussion}

The best results in terms of getting high quality of parametric images are for high thermal gradients. Results of reconstruction is shown on Fig. 3. High thermal gradient is needed for images registration in sequence to establish sub pixel shifting. Sometimes the special markers are necessary during capturing thermal images.

These preliminary findings show that the proposed methodology is able to determine the small structural changes. Application of modern IR-thermography equipment, of improved parameters comparing to the camera used in this study (spatial and thermal resolutions, acquisition speed), will improve the quality of differentiation and evaluation of indicated regions of tested living organs. Still, this requires additional processing to improve the quality of parametric images [13]. Since thermography is based on surface measurements it is relatively simply to use surface markers (e.g. reference signs on the tissue surface with a special pen or a marker and then normalize all images in the series to the frame of reference - usually to the initial image).

HR image reconstruction procedure is very sensitive to errors in the phase of recording low-resolution image sequences. So important is the introduction of camera shake to ensure the displacement sub pixels. In addition, care must be taken to monitoring of the patient did not move during the test because the elimination of movement is another computationally expensive task, which may introduce additional errors of estimation, which is reflected in the quality of reconstruction. Incorrect reconstruction results shown in Figure 4 You can see a clear shift in the lower part of the thermogram - the edge of the a bra of the patient.
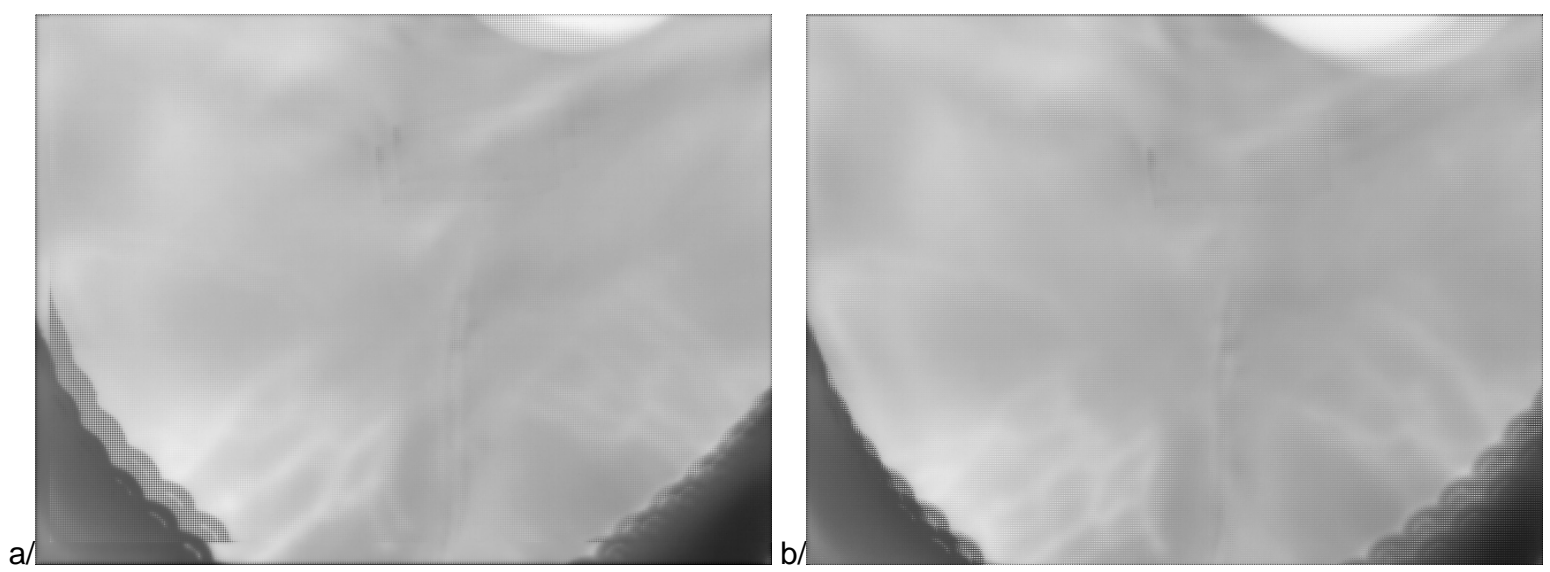

Fig. 4. Example o HR reconstruction, factor $2 x ; a /$ Vandewalle algorithm and POCS, b/ Iterative Taylor and POCS algorithm

However keep in mind that even doubling the size of the image gives a substantial, tangible benefits and it is very desirable from the standpoint of used imaging systems. Available commercial solutions usually offer higher resolution. Twice increasing matrix corresponds to the use of four times the amount of radiation detectors. In other words, this is equivalent to using a matrix of four times more pixels. This is contrary to appearances, a very good result, especially in the context of thermography when considering low resolution cameras on the market and their high cost on a per pixel generated image. It is also worth to remember that with the way the camera with a relatively high-resolution matrix can be reconstructed using the Super-Resolution obtained image size is not available using the currently available cameras. However there are new thermal cameras offering embedded HR algorithm [13].

It was observed that Superresolution did give an edge over bicubic interpolation, and can be used in practical scenario. Though there are three points that should be kept in mind:

1. Image Registration is extremely essential to successful Superresolution.

2. If the low-res images have redundant data, then Superresolution does not work very well.

3. If the low-res images have all the information about the high-res, i.e. they have zero redundant data among them, then Superresolution works perfectly

One important fact about the algorithms used in this project is that they don't require tweaking of many parameters, so same code works well for a wide range of images.

This project restricted the motion between images to be global. In real-life we seldom have global motion and will mostly have multiple motions between frames, but this project still gives a very good intuition into the complexity of Superresolution and different ways to solve it. 


\section{REFERENCES}

[1] Maldague X.P., Theory and practice of infrared technology for non-destructive testing, J. Wiley \& Sons, Inc., New York, 2001.

[2] Wu D., Salerno A., Schonbach B., Halin H., Busse G., Phase sensitive modulation thermography and its applications for NDE, Thermosense XIX, SPIE Proc. 3056, 1997, pp. 176-183.

[3] Kaczmarek M., Nowakowski A., Suchowirski M., Siebert J., Stojek W., Active dynamic thermography in cardiosurgery, QIRT Journal, Vol. 4, No 1/2007, 2007, pp. 107-123.

[4] Parrk S. C., Park M. K., Kang M. G., "Super-resolution image reconstruction: a technical overview", IEEE Signal Processing Magazine, No 20(3), pp. 21-36, May 2003.

[5] Chikamatsu S., et al, "Super-resolution Technique for Thermography with Dual-Camera System", Proceedings of 2010 IEEE International Symposium on Circuits and Systems (ISCAS), 2010, pp. 1895-1898.

[6] Vandewelle P., Süsstrunk S., Vetterli M., A Frequency Domain Approach to Registration of Aliased Images with Application to Super-resolution, EURASIP Journal on Applied Signal Processing, 2006, pp. 1-14

[7] Argyriou V., Vlachos T., On the estimation of subpixel motion using phase correlation, Journal of Electronic Imaging, vol 16, no 3, pp. 033018-1 - 033018-8, 2007.

[8] Keren D., Peleg S., Brada R., Image Sequence Enhancement Using Sub-pixel Displacements, IEEE Computer Society Conference on Computer Vision and Pattern Recognition, Ann Arbor, Michigan, USA, 1988, pp. 742746.

[9] Haik O., Yitzhaky Y., Superresolution reconstruction of a video captured by a vibrated time delay and integration camera, Journal of Electronic Imaging, vol 15(2), no 023006, 2006

[10] Chatterjee P., Mukherjee S., Chaudhuri S., Seetharaman G., Application of Papoulis-Gerchberg Method in Image Super-resolution and Inpainting, The Computer Journal Vol. 00 No. 0, Oxford University Press, 2007

[11] Zomet A., Rav-Acha A., Peleg S., Robust Super-Resolution, Proceedings of the International Conference on Computer Vision and Pattern Recognition (CVPR), Hawaii, December 2001. 2001.

[12] Panagiotopoulou A., Anastassopoulos V., Super-Resolution Reconstruction of Thermal Infrared Images, Proceedings of the 4th WSEAS International Conference on REMOTE SENSING, 2008, pp.40-44.

[13] Testo SuperResolution - the patent-pending technology for high-resolution thermal images, http, http://www.ferret.com.au/c/testo/Testo-SuperResolution-patent-pending-technology-for-high-resolution-thermalimages-n1828762. 